\title{
Oxyresveratrol and its synthetic derivatives on the stimulation of glucose uptake in skeletal muscle cells and the activation of AMPK
}

\author{
Tharita Kitisripanya $^{\mathrm{a}}$, Hathairat Buraphaka ${ }^{\mathrm{b}}$, Panitch Boonsnongcheep ${ }^{\mathrm{c}}$, Boonchoo Sritularak ${ }^{\mathrm{d}}$, \\ Kittisak Likhitwitayawuid ${ }^{\mathrm{d}}$, Waraporn Putalun ${ }^{\mathrm{b}, *}$ \\ a Faculty of Pharmacy, Mahidol University, Bangkok 10400 Thailand \\ b Faculty of Pharmaceutical Sciences, Khon Kaen University, Khon Kaen 40002 Thailand \\ c Institute of Molecular Biosciences, Mahidol University, Nakhon Pathom 73170 Thailand \\ ${ }^{d}$ Faculty of Pharmaceutical Sciences, Chulalongkorn University, Bangkok 1033 Thailand
}

*Corresponding author, e-mail: waraporn@kku.ac.th

Received 28 Mar 2021

Accepted 22 Sep 2021

\begin{abstract}
Diabetes mellitus (DM) is a hyperglycemic condition occurring due to insulin resistance or impaired insulin secretion. Structural modifications of natural products are a promising way to discover new lead compounds for DM management. Oxyresvertrol, abundantly found in Artocarpus lacucha Buch. - Ham. heartwood and Morus alba L. wood, exhibited biological activities against metabolic syndromes associated with diseases including DM. Hence, in this study, oxyresveratrol was used as the precursor for its synthetic derivatives. Then, the compound and its nineteen derivatives were investigated for their glucose uptake activities in L6 rat skeletal muscle cells. The results showed that oxyresveratrol and its derivative, 5-carboxy-2,3',4,5'-tetrahydroxystilbene, possess a higher glucose uptake activity than metformin. They also elevated AMPK alpha-1 mRNA expression in a dose-dependent manner. Moreover, their binding energy against AMPK active sites was reported, and the finding would be applicable to further investigations of oxyresveratrol, including structure-activity relationship and structural modification.
\end{abstract}

KEYWORDS: oxyresveratrol, medicinal chemistry, glucose uptake, AMPK, molecular docking analysis

\section{INTRODUCTION}

Diabetes mellitus (DM) is a hyperglycemic condition occurring due to insulin resistance or impaired insulin secretion. One of the pathological causes of DM was developed from metabolic syndromes which are associated with hypertension, hyperglycemia, high belly fat accumulation, and abnormality of cholesterol and triglyceride levels in blood circulation. These conditions lead to complications in DM patients, e.g., cardiovascular disease, ischemic stroke, life-threatening severe renal failure [1]. Hence, the therapeutic approach based on the mechanism involving metabolic syndromes would be the prevention or treatment intervention of DM.

The AMP-activated protein kinase (AMPK) is an alpha-beta-gamma $(\alpha-\beta-\gamma)$ heterotrimer found in various cell types. It plays an essential role in regulating whole-body glucose homeostasis. In recent years, AMPK activators, based on theirs reported actions in the liver and the skeletal muscle, have been known as a promising pharmacological target for the prevention and the treatment of type $2 \mathrm{DM}$ and metabolic diseases [2,3]. The catalytic subunit of the AMPK, including the AMPK $\alpha 1$ and the AMPK $\alpha 2$ sub-types, is the $\alpha$ subunit. While the AMPK $\alpha 1$ is generally distributed in the body, the AMPK $\alpha 2$ is highly expressed in heart, liver, and skeletal muscles. In the skeletal muscles, the AMPK $\alpha 2$ sub-type constitutes more than half of the total AMPK $\alpha$ sub-type [4]. This implies that the AMPK $\alpha 2$ plays an essential metabolic role in the skeletal muscles. Both the AMPK $\alpha 1$ and the AMPK $\alpha 2$ subtypes are critical regulators of glucose metabolism with different functions. In DM patients, there is a malfunction of glucose uptake in skeletal muscle cells and adipocytes. Therefore, investigations of natural products possessing glucose uptake activity as potential agents could be applied for the prevention or the relief of insulin resistance. Besides, the knowledge of phytochemicals profiles in plant species could be a strategy to find the potential sources of active compounds against DM [5]. Several compounds including metformin [6], polyphenols (such as resveratrol) [7], quercetin [8], and ros- 
marinic acid [9] are also recognized for activating AMPK and increasing muscle glucose uptake.

Oxyresveratrol (trans-2, 3', 4, 5' -tetrahydroxystilbene) is a natural stilbenoid isolated from Artocarpus lacucha Buch. - Ham. heartwood and abundantly found in mulberry wood (Morus alba L.). It has parallel biological effects of resveratrol, which include anti-oxidative, antiviral, anti-inflammatory, and neuroprotective activities [10-15]. Besides, the effects of oxyresveratrol on mechanisms associated with metabolic syndromes have been recently investigated, for example, the alpha glucosidase inhibitory activity [16], the amelioration of obesityassociated symptoms [17], the hepatoprotective function in non-alcoholic fatty liver disease [18], the prevention of obesity, and the improvement of glucose metabolism $[19,20]$. To determine the potential of oxyresveratrol and its synthetic derivatives, their effects on glucose uptake activity (which is associated with anti-diabetic activity) in skeletal muscle cells were investigated. Consequently, high potent compounds were selected for AMPK expression. In addition, to explore the structure-activity relationship of the oxyresveratrol and its derivatives on AMPK, their simulated binding energies were investigated using molecular docking analysis. The results from these investigations could be applied for further structural modification of oxyresveratrol as a potential anti-diabetic agent for DM therapy.

\section{MATERIALS AND METHODS}

Chemicals. $\alpha$-MEM, fetal bovine serum (FBS), and penicillin-streptomycin $(10000 \mathrm{IU} / \mathrm{ml})$ were purchased from Thermo Fisher Scientific (Grand Island, NY, USA). Glucose (GO) assay kit, sodium dodecyl sulfate (SDS), metformin, and 3-(4, 5dimethyl thiazol-2-yl)-5-diphenyl tetrazolium bromide (MTT) were obtained from Sigma Aldrich (St. Louis, MO, USA). Insulin $(100 \mathrm{IU} / \mathrm{ml})$ was obtained from Biocon (Bangalore, India). All other chemicals used were of analytical grade.

\section{PREPARATION OF THE SYNTHETIC DERIVATIVES}

Oxyresveratrol was isolated from heartwood of $A r$ tocarpus lacucha Buch. - Ham. as previously described [13]. Nineteen derivatives of oxyresveratrol were synthesized based on the earlier method [16] using oxyresveratrol as a precursor. O-alkylation, acylation, halogenation, and aromatic electrophilic substitution were used as the synthetic strategies for preparation of oxyresveratrol derivatives. For example, in $O$-alkylation, oxyresveratrol was allowed to

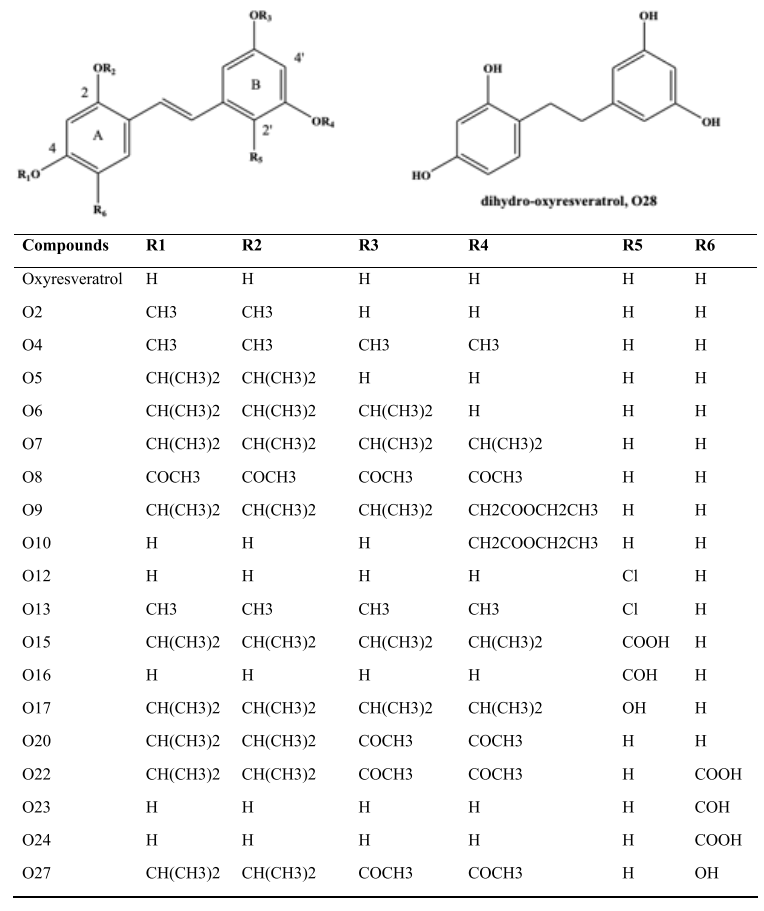

Fig. 1 Chemical structures of oxyresveratrol and its synthetic derivatives.

react with methyl iodide in acetone at room temperature to obtain di-, and tetra- $\mathrm{O}$-methylated as $\mathrm{O} 2$ and $\mathrm{O} 4$, respectively. The alkylation of oxyresveratrol with 2-bromopropane $/ \mathrm{K}_{2} \mathrm{CO}_{3}$ in $\mathrm{DMF}$ gave the corresponding di-, tri-, and tetra-O-isopropyl compounds as 05,06 , and 07 , respectively. The chemical structures of oxyresveratrol and the synthetic derivatives are shown in Fig. 1.

Cell lines and culture medium. L6 (rat skeletal muscle, ATCC ${ }^{\circledR}$ CRL-1458) cell culture was purchased from the American Type Culture Collection (Manassas, VA, USA). Stock cells of L6 were cultured in a growth medium consisting of $\alpha$-MEM, $10 \%$ FBS, and $1 \%$ penicillin-streptomycin at $37{ }^{\circ} \mathrm{C}$ under $5 \%$ $\mathrm{CO}_{2}$.

Cytotoxicity. The cytotoxicity test was performed at $24 \mathrm{~h}$ after the cell treatment following Riss et al [21] with some modification. The medium was adjusted to $200 \mu \mathrm{l}$ per well. The cells were treated with $20 \mu \mathrm{l}$ MTT solution $(5 \mathrm{mg} / \mathrm{ml})$ and incubated at $37^{\circ} \mathrm{C}$ under $5 \% \mathrm{CO}_{2}$ for $2 \mathrm{~h}$. To dissolve the formazan crystal, $200 \mu 1$ solubilization solution (40\% DMF, $2 \%$ glacial acetic acid, and 16\%w/v SDS in distilled water) was added to each well, and the well was shaken for $20 \mathrm{~min}$. Then, the supernatants were collected to measure the absorbance at $595 \mathrm{~nm}$ using model 550 microplate reader (Biorad, Her- 
cules, United States). The cytotoxicity was shown as the percentage of cell viability.

Glucose-uptake assay. The glucoseuptake assay was performed following Inthongkaew et al [22]. Briefly, rat L6 myoblasts were maintained in $\alpha$-MEM supplemented with $10 \% \mathrm{FBS}$ and $1 \%$ penicillin-streptomycin at $37^{\circ} \mathrm{C}$ under $5 \% \mathrm{CO}_{2}$. The cells were then added in 24well plates at a density of $2 \times 10^{4}$ cells/well. Once the cells reached $90 \%$ confluence, the medium was switched to $\alpha$-MEM with $2 \%$ FBS and $1 \%$ penicillinstreptomycin, the differentiated medium. The cells were allowed to differentiate into myotubes for 5-7 days, and the medium was changed every other day. After that, the myotubes were incubated at $37^{\circ} \mathrm{C}$ under $5 \% \mathrm{CO}_{2}$ for $24 \mathrm{~h}$ with various concentrations (1, 10 and $100 \mu \mathrm{g} / \mathrm{ml}$ ) of oxyresveratrol and the nineteen derivatives. The positive controls were metformin $(2 \mathrm{mM})$ and insulin $(500 \mathrm{nM})$. The differentiated medium plus $0.1 \%$ DMSO was used as the diluent and the negative control. Then, the medium was collected, and the glucose level was determined using a glucose oxidase assay kit. The glucose uptake of test compounds was presented as the relative ratio over the percentage of glucose uptake of the negative control.

Expression levels of the AMPK $\alpha 1$ and the AMPK $\alpha 2$ affected by oxyresveratrol and its derivatives. Cells were culture in 24-well plates in a similar manner as previously described. After differentiation, they were treated with 1,10 , and $100 \mu \mathrm{g} / \mathrm{ml}$ oxyresveratrol and $\mathrm{O} 24$ for $24 \mathrm{~h}$. Metformin $(2 \mathrm{mM})$ and insulin $(500 \mathrm{nM})$ were used as positive controls. The differentiate medium plus $0.1 \%$ DMSO was used as the diluent and the negative control. Then, the medium was removed and total RNA was extracted using TRIzol following the manufacturer's protocol. The extracted RNA was converted to cDNA with the ReverTraAce Q PCR RT Master Mix (Toyobo, Osaka, Japan) following the recommended protocol. The real-time PCR analysis was performed using SYBR Green PCR Master Mix (Bio-Rad) in a CFX96 Real-Time PCR detection system (Bio-Rad). GADPH gene was used as a reference. Primers for amplification of the AMPK $\alpha 1$, the AMPK $\alpha 2$, and the GADPH are shown in Table S1. The PCR conditions were as follow: 3 min of $95^{\circ} \mathrm{C}$ initial denaturation; followed by 40 cycles of $20 \mathrm{~s}$ at $95^{\circ} \mathrm{C}, 20 \mathrm{~s}$ at $56^{\circ} \mathrm{C}$, and $30 \mathrm{~s}$ at $72^{\circ} \mathrm{C}$. Results are reported as the fold expression change relative to the control group. The experiments were conducted at least in triplicate. Experimental results were expressed as means \pm standard deviations. Multiple group comparisons were performed with one-way ANOVA with Dunnett $t$-test at $p<0.05$ using SPSS (version 17.0).

Docking analysis. The three-dimensional structure of AMPK was retrieved from Protein Data Bank (PDB code: 4CFE) and imported into the AutoDockTools (ADT, version 1.5.6). All water and hydrogen molecules were removed and added from the protein, respectively. The co-crystalline ligand, a benzimidazole derivative (991), was an AMPK activator and used as a reference compound [23]. The three-dimensional (3-D) structures of ligands were drawn using Chem3D Ultra 8.0, saved as pdb formats, and then changed into pdbqt formats by ADT. All water and hydrogen molecules were also removed and added from the ligands, respectively. Then, Gasteiger charges were computed. The receptor grid box for docking size was centered on the ligand in the active site on the $\alpha-2$ subunit of the AMPK and set to 40, 40, 40 number of $x-, y-, z$ grid points and grid center at 476.564, 41.951, and 987.875 of $x-, y-$, and $z$-coordinates with a spacing of $0.375 \AA$ using the AutoGrid 4.0. Each compound was docked inside a grid for ten conformations by AutoDock 4.0. Other docking parameters were set as default values [24]. The AutoDock docking runs were performed on an Intel ${ }^{\circledR}$ Core $^{\mathrm{TM}} \mathrm{CPU}$ i5$7200 \mathrm{U} 2.50 \mathrm{GHz}$ processor with $4 \mathrm{~GB}$ RAM running Windows (Windows10, 64-bit) operating system. After molecular docking, the lowest binding energy of each ligand was selected and compared with the the 991 and the metformin.

\section{RESULTS AND DISCUSSION}

\section{Cytotoxicity}

After the exposure to oxyresveratrol and its derivatives at various concentrations of 1,10 and $100 \mu \mathrm{g} / \mathrm{ml}$, the percentage of cell viability in all treatments exhibited no cytotoxicity against L6 myotubes except $\mathrm{O} 2, \mathrm{O} 5, \mathrm{O}, \mathrm{O} 10, \mathrm{O} 12$, and 016 derivatives at a concentration of $100 \mu \mathrm{g} / \mathrm{ml}$ with less than $80 \%$ cell viability (Fig. $2 \mathrm{~A}$ ).

\section{Glucose-uptake assay}

The amount of glucose uptake in L6 myotubes was an indirect measurement of glucose content in the treated cell medium after $24 \mathrm{~h}$ exposure to the test compounds. In Fig. 2B, the amount of glucose uptake was shown as fold-over basal. The positive controls, metformin and insulin, exhibited the uptakes of 1.74-fold and 1.28-fold, respectively, whereas the oxyresveratrol and its derivatives $(\mathrm{O} 22$, 

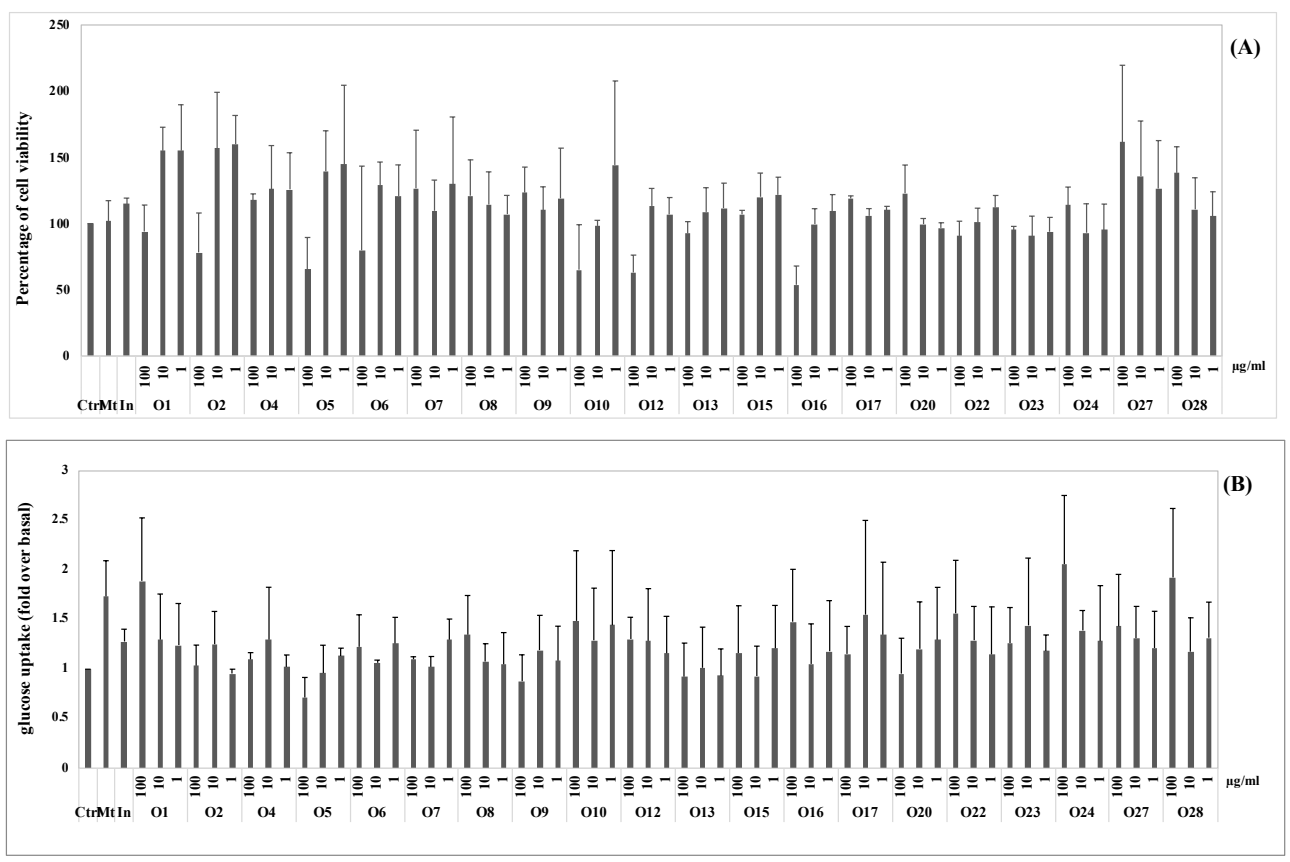

Fig. 2 Percentage of cell viability (A) and glucose uptake activity (B) of the control (ctrl), the metformin (Mt, $2 \mathrm{mM}$ ), the insulin (In, $500 \mathrm{nM}$ ), the oxyresveratrol (O1), and the derivatives at concentrations of 1, 10, and $100 \mu \mathrm{g} / \mathrm{ml} \mathrm{in}$ the L6 skeletal muscle cells after 24 h of exposure (mean \pm SD, $n=9$ ).

O24, and O28) at concentration of $100 \mu \mathrm{g} / \mathrm{ml}$ showed glucose uptake higher than 1.5-fold (1.89, $1.56,2.06$, and 1.92-fold, respectively). Hence, O24, which exhibited the highest glucose uptake activity, and oxyresveratrol were selected for the AMPK expression experiment.

\section{Expression of AMPK $\alpha 1$ and AMPK $\alpha 2$}

Activation of AMPK resulted in an increase of glucose uptake in muscle cells, and it was an effective therapeutic strategy for diabetics [25]. The determination of AMPK mRNA expression in L6 myotubes treated with oxyresveratrol and the $\mathrm{O} 24$ derivative can reveal the mechanism associated with the AMPK-dependent pathway. In Fig. 3, both the oxyresveratrol and the $\mathrm{O} 24$ derivative significantly up-regulated the AMPK $\alpha 1$ and the AMPK $\alpha 2$ expression levels when compared with the control. The transcript expression levels of both AMPK subunits showed a similar pattern with glucose uptake in a dose-dependent manner, except for the $100 \mu \mathrm{g} / \mathrm{ml}$ oxyresveratrol in $\alpha 2$ subunit. It can be explained that the AMPK $\alpha 1$ takes part in the regulation of glucose uptake but not the AMPK $\alpha 2$ $[26,27]$. Therefore, these results suggest that the increase in glucose uptake affected by oxyresveratrol and $\mathrm{O} 24$ derivative may be correlated with the

\section{AMPK signaling pathway.}

\section{Docking analysis}

The structure-activity relationship of oxyresveratrol and its derivatives on AMPK was investigated by molecular docking analysis. The 3-D structures of oxyresveratrol and the nineteen derivatives binding at the active binding site of 991 (the reference compound) on the AMPK were simulated (Fig. S1) [23]. The lowest binding energy (LBE) values of individual compounds were in the range of -8.33 to $-6.20 \mathrm{kcal} / \mathrm{mol}$, while the LBE of 991 was $-10.66 \mathrm{kcal} / \mathrm{mol}$ (Fig. 4). To assess the high glucose uptake activity compounds in this study, the LBE values of oxyresveratrol, $\mathrm{O} 22, \mathrm{O} 24$, and O28, which respectively were $-6.32,-6.20,-7.46$, and $-6.23 \mathrm{kcal} / \mathrm{mol}$ were analyzed. The LBE could be used to predict the binding interaction of compounds or ligands against the binding site. The high glucose uptake activity could be from the carboxyl $(-\mathrm{COOH})$ substitute in $\mathrm{R} 6$ position as in $\mathrm{O} 22$ and O24. Besides, the hydrogen $(-\mathrm{H})$ substitutes in $\mathrm{R} 1$ to R5 positions may contribute in less interaction of ligands to the binding site (higher LBE values in oxyresveratrol and O28) without relating to the glucose uptake activity. In general, the lower the LBE is, the more easily a compound binds to an 

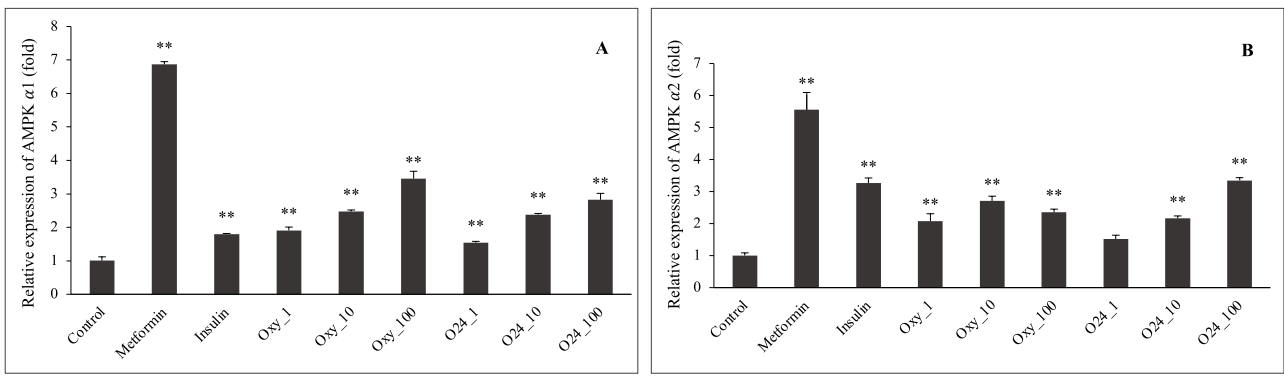

Fig. 3 Expression levels of AMPK $\alpha 1$ (A) and AMPK $\alpha 2$ (B) in L6 myotube cells after incubation with tested compound for 24 h. Data are expressed as means $\pm \mathrm{SD}$, and $* * p<0.05$ compared with control by one-way ANOVA, Dunnett $t$-test.

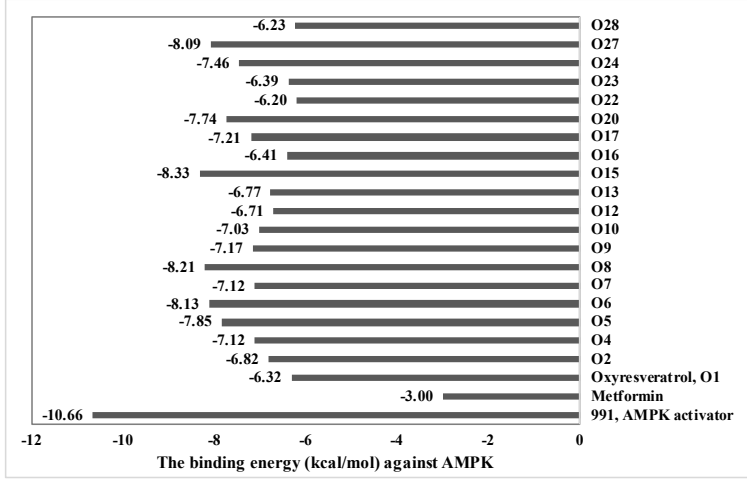

Fig. 4 Binding energy against AMPK of AMPK activator (991), metformin, oxyresveratrol, and the derivatives.

active site. This principle was not applicable to AMPK that possesses other active sites associated with metabolic diseases [23]. Despite the high potency of metformin in glucose uptake assay and the elevated AMPK mRNA expression, its LBE was the highest representing the lowest affinity to the AMPK active site. The result conformed to the effect of metformin acting via a non-AMPK pathway to promote glucose uptake in the skeletal muscle cells $[6,28]$. Consequently, the effects of oxyresveratrol and the nineteen derivatives could not be predicted solely on AMPK interaction, especially in an active site like the compound 991's, by using computational modeling.

\section{CONCLUSION}

The effects of oxyresveratrol and its derivatives on glucose uptake activity were investigated. The structural modification of oxyresveratrol by substituted carboxyl on C-5 of ring A (compound O24, 5-carboxy-2,3' $4,5^{\prime}$-tetrahydroxystilbene) not only increased glucose uptake activity in skeletal muscle cells in a dose-dependent manner but also elevated
AMPK mRNA expression. Moreover, the results from molecular docking analysis revealed that the binding energy was not much correlated to the glucose uptake activities. However, the binding energy on the binding site of test compounds can be used to predict the conformation which could be applied to further investigations including structure-activity relationship and structural modification.

\section{Appendix A. Supplementary data}

Supplementary data associated with this article can be found at http://dx.doi.org/10.2306/ scienceasia1513-1874.2021.107.

Acknowledgements: This work was support by the Program Management Unit for Human Resources and Institutional Development, Research and Innovation (PMU-B) from the Office of National Higher Education Science Research and Innovation Policy Council, Thailand.

\section{REFERENCES}

1. Rochlani Y, Pothineni NV, Kovelamudi S, Mehta JL (2017) Metabolic syndrome: pathophysiology, management, and modulation by natural compounds. Ther Adv Cardiovasc Dis 11, 215-225.

2. Ruderman NB, Carling D, Prentki M, Cacicedo JM (2013) AMPK, insulin resistance, and the metabolic syndrome. J Clin Invest 123, 2764-2772.

3. Miglianico M, Nicolaes GAF, Neumann D (2016) Pharmacological targeting of AMP-activated protein kinase and opportunities for computer-aided drug design. J Med Chem 59, 2879-2893.

4. Stapleton D, Mitchelhill KI, Gao G, Widmer J, Michell BJ, Teh T, House CM, Fernandez CS, et al (1996) Mammalian AMP-activated protein kinase subfamily. J Biol Chem 271, 611-614.

5. Choonong R, Sermpradit W, Kitisripanya T, Sritularak B, Putalun W (2019) The contents of bibenzyl derivatives, flavonoids and a phenanthrene in selected Dendrobium spp. and the correlation with their antioxidant activity. ScienceAsia 45, 245-252. 
6. Turban S, Stretton C, Drouin O, Green CJ, Watson ML, Gray A, Ross F, Lantier L, et al (2012) Defining the contribution of AMP-activated protein kinase (AMPK) and protein kinase $C$ (PKC) in regulation of glucose uptake by metformin in skeletal muscle cells. J Biol Chem 287, 20088-20099.

7. Breen DM, Sanli T, Giacca A, Tsiani E (2008) Stimulation of muscle cell glucose uptake by resveratrol through sirtuins and AMPK. Biochem Biophys Res Commun 374, 117-122.

8. Dhanya R, Arya AD, Nisha P, Jayamuthy P (2017) Quercetin, a lead compound against type 2 diabetes ameliorates glucose uptake via AMPK pathway in skeletal muscle cell line. Front Pharmacol 8, ID 336.

9. Vlavcheski F, Naimi M, Murphy B, Hudlicky T, Tsiani E (2017) Rosmarinic acid, a rosemary extract polyphenol, increases skeletal muscle cell glucose uptake and activates AMPK. Molecules 22, ID 1669.

10. Chung KO, Kim BY, Lee MH, Kim YR, Chung HY, Park JH, Moon JO (2003) In-vitro and in-vivo antiinflammatory effect of oxyresveratrol from Morus alba L. J Pharm Pharmacol 55, 1695-1700.

11. Lorenz P, Roychowdhury S, Engelmann M, Wolf G, Horn TF (2003) Oxyresveratrol and resveratrol are potent antioxidants and free radical scavengers: effect on nitrosative and oxidative stress derived from microglial cells. Nitric Oxide 9, 64-76.

12. Likhitwitayawuid K, Sritularak B, Benchanak K, Lipipun V, Mathew J, Schinazi RF (2005) Phenolics with antiviral activity from Millettia erythrocalyx and Artocarpus lakoocha. Nat Prod Res 19, 177-182.

13. Chuanasa T, Phromjai J, Lipipun V, Likhitwitayawuid K, Suzuki M, Pramyothin P, Hattori M, Shiraki K (2008) Antiherpes simplex virus (HSV-1) activity of oxyresveratrol derived from Thai medicinal plant: mechanism of action and therapeutic efficacy on cutaneous HSV-1 infection in mice. Antiviral Res 80, 62-70.

14. Aftab N, Likhitwitayawuid K, Vieira A (2010) Comparative antioxidant activities and synergism of resveratrol and oxyresveratrol. Nat Prod Res 24, 1726-1733.

15. Weber JT, Lamont M, Chibrikova L, Fekkes D, Vlug AS, Lorenz P, Kreutzmann P, Slemmer JE (2012) Potential neuroprotective effects of oxyresveratrol against traumatic injury. Eur $J$ Pharmacol 680, 55-62.

16. Chatsumpun N, Chuanasa T, Sritularak B, Lipipun V, Jongbunprasert V, Ruchirawat S, Ploypradith P, Likhitwitayawuid K (2016) Oxyresveratrol: structural modification and evaluation of biological activities. Molecules 21, ID 489.

17. Tan HY, Tse IM, Li ET, Wang M (2017) Oxyresveratrol supplementation to $\mathrm{C} 57 \mathrm{bl} / 6$ mice fed with a high-fat diet ameliorates obesity-associated symptoms. Nutrients 9, ID 147.

18. Lee JH, Baek SY, Jang EJ, Ku SK, Kim KM, Ki SH, Kim
CE, Park KI, et al (2018) Oxyresveratrol ameliorates nonalcoholic fatty liver disease by regulating hepatic lipogenesis and fatty acid oxidation through liver kinase B1 and AMP-activated protein kinase. Chem Biol Interact 289, 68-74.

19. Choi JH, Song NJ, Lee AR, Lee DH, Seo MJ, Kim S, Chang SH, Yang DK, et al (2018) Oxyresveratrol increases energy expenditure through foxo3amediated ucp1 induction in high-fat-diet-induced obese mice. Int J Mol Sci 20, ID 26.

20. Pan $\mathrm{MH}$, Koh YC, Lee TL, Wang B, Chen WK, Nagabhushanam K, Ho CT (2019) Resveratrol and oxyresveratrol activate thermogenesis via different transcriptional coactivators in high-fat diet-induced obese mice. J Agric Food Chem 67, 13605-13616.

21. Riss TL, Moravec RA, Niles AL, Benink HA, Worzella TJ, Minor L (2004) Cell viability assays. In: Markossian S, et al (eds) Assay Guidance Manual, Eli Lilly \& Company and the National Center for Advancing Translational Sciences, USA, pp 317-342.

22. Inthongkaew P, Chatsumpun N, Supasuteekul C, Kitisripanya T, Putalun W, Likhitwitayawuid K, Sritularak B (2017) Alpha-glucosidase and pancreatic lipase inhibitory activities and glucose uptake stimulatory effect of phenolic compounds from Dendrobium formosum. Rev Bras Farmacogn 27, 480-487.

23. Xiao B, Sanders MJ, Carmena D, Bright NJ, Haire LF, Underwood E, Patel BR, Heath RB, et al (2013) Structural basis of AMPK regulation by small molecule activators. Nat Commun 4, ID 3017.

24. Morris GM, Huey R, Lindstrom W, Sanner MF, Belew RK, Goodsell DS, Olson AJ (2009) Autodock4 and AutoDockTools4: automated docking with selective receptor flexibility. J Comput Chem 16, 2785-2791.

25. Kjøbsted R, Pedersen AJ, Hingst JR, Sabaratnam R, Birk JB, Kristensen JM, Højlund K, Wojtaszewski JF (2016) Intact regulation of the AMPK signaling network in response to exercise and insulin in skeletal muscle of male patients with type 2 diabetes: illumination of AMPK activation in recovery from exercise. Diabetes 65, 1219-1230.

26. Jensen TE, Schjerling P, Viollet B, Wojtaszewski JF, Richter EA (2008) AMPK alpha1 activation is required for stimulation of glucose uptake by twitch contraction, but not by $\mathrm{H}_{2} \mathrm{O}_{2}$, in mouse skeletal muscle. PLoS One 3, e2102.

27. Maarbjerg SJ, Jorgensen SB, Rose AJ, Jeppesen J, Jensen TE, Treebak JT, Birk JB, Schjerling P, et al (2009) Genetic impairment of (alpha)2-AMPK signaling does not reduce muscle glucose uptake during treadmill exercise in mice. Am J Physiol Endocrinol Metab 297, E924-E934.

28. Madiraju AK, Erion DM, Rahimi Y, Zhang XM, Braddock DT, Albright RA, Prigaro BJ, Wood JL, et al (2014) Metformin suppresses gluconeogenesis by inhibiting mitochondrial glycerophosphate dehydrogenase. Nature 510, 542-546. 


\section{Appendix A. Supplementary data}

Table S1 Primers used in the real-time PCR reactions.

\begin{tabular}{lll}
\hline Target gene & Forward primers $\left(5^{\prime}\right.$ to $\left.3^{\prime}\right)$ & Reverse primers $\left(5^{\prime}\right.$ to $\left.3^{\prime}\right)$ \\
\hline AMPK $\alpha 1$ & ATC CGC AGA GAG ATC CAG AA & CGT CGA CTC TCC TTT TCG TC \\
AMPK $\alpha 2$ & TGA GGT GGT GGA GCA GAG G & TGC TGC CAG TCA AAG AAC CA \\
GADPH (reference) & GAA GGT CGG TGT GAA CGG AT & ACC AGC TTC CCA TTC TCA GC \\
\hline
\end{tabular}

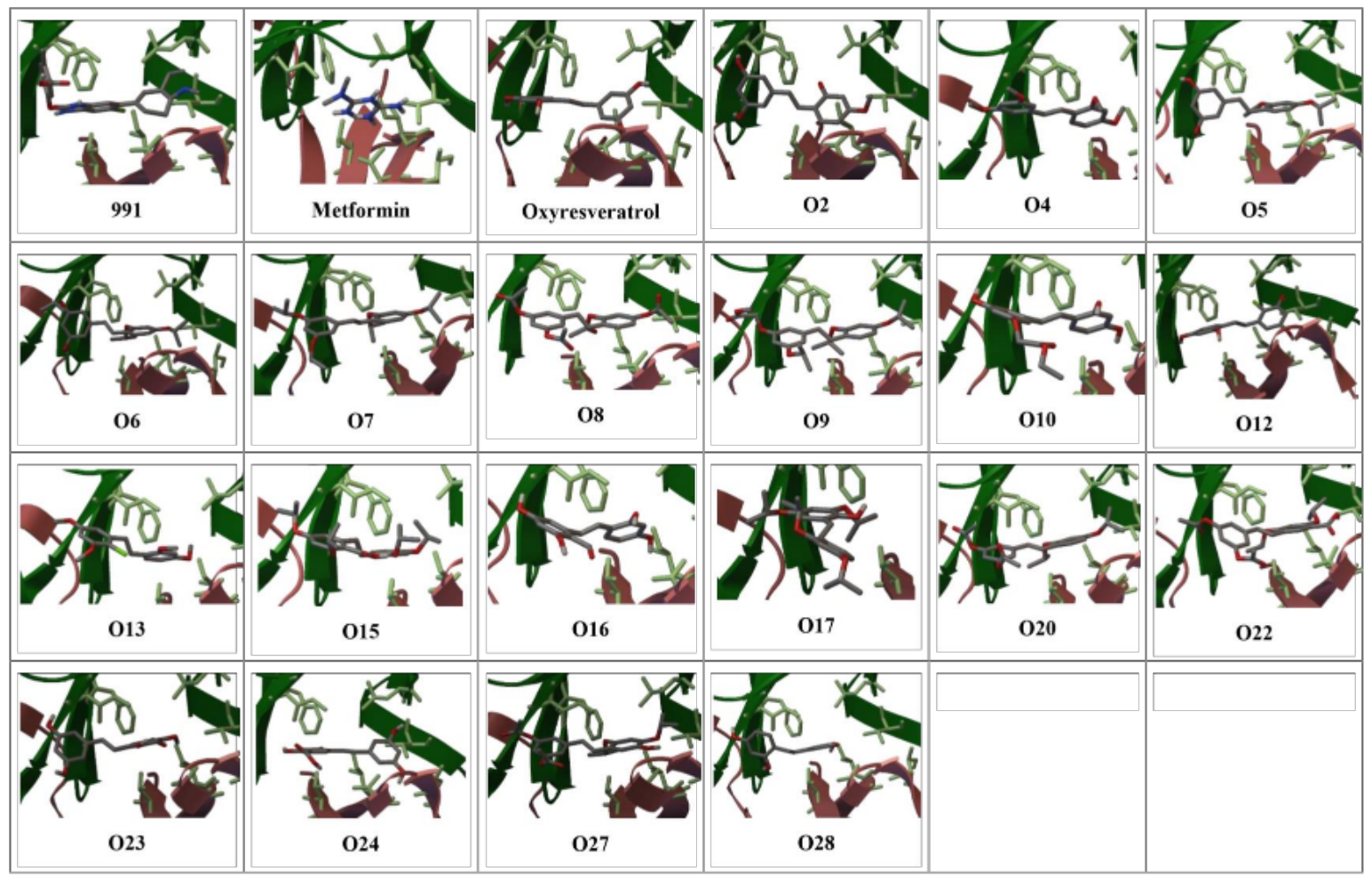

Fig. S1 Simulation of AMPK activator (991), metformin, oxyresveratrol and its derivatives on AMPK, based on 991 compound active binding site. 